\title{
INTRALESIONAL LOW DOSE METHYLPREDNISOLONE FOR THE TREATMENT OF ACTIVE PHASE PEYRONIE'S DISEASE: A SINGLE CENTER, PRELIMINARY PROSPECTIVE NON-RANDOMISED STUDY
}

iyimser $\ddot{u ̈ r}^{1}$ and ata özen ${ }^{1}$

${ }^{1}$ Eskişehir Osmangazi Üniversitesi Tıp Fakültesi

September 11, 2020

\begin{abstract}
Aim There are few types of drugs that can be used in the active phase of Peyronie's disease. Methylprednisolone is a corticosteroid with a strong anti-inflammatory effect. In this study, we aimed to evaluate the effect of intralesional low dose methylprednisolone treatment on patients in the active phase of Peyronie's disease. Patients and Methods Forty-eight patients suffering from Peyronie's disease active phase symptoms were included in the study. Methylprednisolone was administered intralesionally for 8 weeks, once per week, at a dose of $40 \mathrm{mg}$. The injection was applied into the plaques, which causes maximum curvature. Patients were evaluated before and after treatment for plaque size, angle of curvature, and erectile dysfunction according to the International Index of Erectile Function-5 and Peyronie's Disease Questionnaire. Results The mean age of the patients was 61.1 (43-78) years. Mean duration of the symptoms was 3.4 (0-9) months. The average plaque size before treatment was $13.6 \mathrm{~mm}$ (7.1-16.8) and after treatment, this value decreased to $10.8 \mathrm{~mm}$ (4.3-14.6) (p:0.025). The average scores of Peyronie's Disease Questionnaire elements; symptom severity, penile pain and bother/discomfort were 12.3, 19.1 and 6.2, respectively before the treatment. These scores were decreased to 8.9, 9.6 and 4.4, respectively after treatment. All subgroups of Peyronie's Disease Questionnaire scores were significantly improved after treatment (p:0.001, p < 0.001, p:0.045, respectively). No adverse events were observed during or after treatment. Conclusion In order to recover the symptoms and signs, new and easily accessible drugs are required for use in the acute period of Peyronie's disease. In this context, treatment with intralesional low dose methylprednisolone in acute phase Peyronie's Disease is a promising and safe treatment option.
\end{abstract}

\section{INTRALESIONAL LOW DOSE METHYLPREDNISOLONE FOR THE TREATMENT OF ACTIVE PHASE PEYRONIE'S DISEASE: A SINGLE CENTER, PRELIMINARY PROSPECTIVE NON-RANDOMISED STUDY}

Running Title: Methylprednisolone for treatment of Peyronie's disease

Dr. Iyimser URE, M.D.*

Dr. Ata OZEN, M.D.*

*Osmangazi University, Faculty of Medicine, Department of Urology, Eskisehir, TURKEY

\section{Corresponding Author:}

Iyimser URE, M.D.

Osmangazi Universitesi, Tip Fakultesi, Uroloji AD

Eskisehir / TURKIYE 
iyimserure@yahoo.com

+90 5303473301

Key Words: Peyronie's disease; Methylprednisolone; Treatment ABSTRACT

Aim

There are few types of drugs that can be used in the active phase of Peyronie's disease. Methylprednisolone is a corticosteroid with a strong anti-inflammatory effect. In this study, we aimed to evaluate the effect of intralesional low dose methylprednisolone treatment on patients in the active phase of Peyronie's disease.

Patients and Methods

Forty-eight patients suffering from Peyronie's disease active phase symptoms were included in the study. Methylprednisolone was administered intralesionally for 8 weeks, once per week, at a dose of $40 \mathrm{mg}$. The injection was applied into the plaques, which causes maximum curvature. Patients were evaluated before and after treatment for plaque size, angle of curvature, and erectile dysfunction according to the International Index of Erectile Function-5 and Peyronie's Disease Questionnaire.

Results

The mean age of the patients was 61.1 (43-78) years. Mean duration of the symptoms was $3.4(0-9)$ months. The average plaque size before treatment was $13.6 \mathrm{~mm}$ (7.1-16.8) and after treatment, this value decreased to $10.8 \mathrm{~mm}$ (4.3-14.6) (p:0.025). The average scores of Peyronie's Disease Questionnaire elements; symptom severity, penile pain and bother/discomfort were 12.3, 19.1 and 6.2 , respectively before the treatment. These scores were decreased to 8.9, 9.6 and 4.4, respectively after treatment. All subgroups of Peyronie's Disease Questionnaire scores were significantly improved after treatment (p:0.001, p $<0.001, \mathrm{p}: 0.045$, respectively). No adverse events were observed during or after treatment.

Conclusion

In order to recover the symptoms and signs, new and easily accessible drugs are required for use in the acute period of Peyronie's disease. In this context, treatment with intralesional low dose methylprednisolone in acute phase Peyronie's Disease is a promising and safe treatment option.

\section{INTRODUCTION}

Peyronie's disease is a condition characterized by the formation of plaque due to collagen accumulation inside the tunica albuginea that surrounds the structures of the penile corpus cavernosum. This plaque can cause deformities such as penile shortening and curvature [1]. Penile pain and discomfort may occur during the active period of the disease, sexual functions are negatively affected, and erectile dysfunction may occur [2]. The incidence of Peyronie's disease was found to be 3.2-8.9\% [3]. Although young men are also affected occasionally, the disease is more common among older men-specifically, those over the age of 45-and its incidence increases with age [4].

Surgical treatment is not recommended in the active period of the disease. In this period, it is aimed to stop the formation of new plaques, avoid the plaque growth, to eliminate the pain. Approaches to treatment include the use of oral treatment agents and intralesional agents [5]. Oral therapies being used today include vitamin E, carnitine, colchicine, potassium amino benzoate, and phosphodiesterase type 5 inhibitors. However, none of these agents' effectiveness has been fully proven [6].

Molecules such as corticosteroids, verapamil, interferon, and collagenase clostridium histolyticum are commonly used in intralesional therapy [5]. Among these, collagenase clostridium histolyticum stands out as the most effective agent, and there are many studies that have proven its effectiveness [1,7-9]. However, in some countries, this drug is difficult to obtain, and the cost of treatment protocols with this drug can be excessive. In addition, collagenase was withdrawn from the European market for commercial reasons. 
Therefore, there is a search for more accessible treatment methods that can be used during Peyronie's active period, especially in developing countries.

Methylprednisolone is a corticosteroid with a strong anti-inflammatory effect. It is used in the treatment of rheumatic diseases, acute/chronic inflammatory diseases, and allergic reactions [10]. It is a fairly inexpensive drug and easy to obtain. Since systemic and local side effects are also rare, it is probably that methylprednisolone could serve as an alternative treatment option in the acute period of Peyronie's disease where inflammatory reactions are at the forefront [11].

The purpose of our study was to investigate the effectiveness and safety of intralesional methylprednisolone treatment in patients presenting in the acute period of Peyronie's disease.

\section{MATERIALS AND METHODS}

\section{Study Design}

This is a prospective non-randomized preliminary study for observing the effectiveness and safety of using intralesional methylprednisolone to treat patients in the active phase of Peyronie's disease. Patients who applied to our clinic for Peyronie's disease and who were in the active phase of the disease between 2018 and 2020 were included in the study. To confirm that the disease was in the acute phase, we used the following criteria for inclusion: (1) less than 12 months passed since the onset of complaints; (2) absence of calcified plaque in penile duplex doppler ultrasound; (3) symptoms of painful erection; and (4) recent change in penile curvature. Patients who had completed the acute phase and progressed to the chronic phase disease stage, who had the presence of calcified plaque, who had prior medical or surgical treatment for Peyronie's disease, who had a history of extra corporeal shock wave therapy (ESWT), who had hourglass and hinge deformity, and who were not sexually active were excluded from the study. Apart from hourglass and hinge deformity, patients with all types of curvature were included in the study.

\section{Pre Treatment work-up}

After erection was achieved by intracavernosal alprostadil injection, the maximum plaque size was measured before and after treatment by performing penile duplex doppler ultrasound in all patients. During the same procedure, the physician investigated whether there was calcified plaque. During erection, curvature angle was measured using a goniometer protractor. The point of injection was determined by marking the maximal curvature point, and the necessity of erection was eliminated before each procedure. To determine the point of maximum curvature, starting at the base of the penis, a straight line was drawn through the center of the straight portion of the penile shaft proximal and distal to the point of maximum curvature. The intersection of these lines was accepted as the maximal point of curvature, and the degree of curvature was determined by measuring the angle between these lines.

Before the first injection and one month after the last injection, all patients were assessed with Peyronie's Disease Questionnaire (PDQ), consisting of 15 questions, and the International Index of Erectile Function (IIEF-5), consisting of 5 questions [12]. The score obtained from questions 1-6 of the PDQ questionnaire evaluates symptom severity (PDQS), questions 7-9 evaluate penile pain (PDQP), and questions 10-15 evaluate bother/discomfort symptoms (PDQB) [13].

\section{Treatment Protocol}

The treatment protocol was designed as an intralesional injection of $40 \mathrm{mg}$ methylprednisolone to the maximum curvature point; this injection was repeated once a week for 8 weeks. No local anesthesia was performed before injection, but patients were allowed to use non-steroidal anti-inflammatory drugs and pain killers after injection. A $23 \mathrm{G}$ needle was used for injection. The dosage was $40 \mathrm{mg}$ of the drug in its pure form (no dilution), which was loaded into a syringe and then inserted into the plaque at a right angle. The drug was then injected at different points of the plaque by slightly moving the needle around the injection point.

During the protocol, treatment was terminated and the patient was excluded from the study in cases of an unexpected side effects or worsening disease symptoms. All adverse events were recorded during the study 
period. All procedures were performed by a single experienced urologist.

All patients voluntarily signed their consent to participate in the study. The study was approved by the Ethics Committee of Eskişehir Osmangazi University Faculty of Medicine.

\section{Statistical Analysis}

Statistical analysis was done with IBM SPSS V23.0 Software. No power analysis were performed. Continuous variables are reported as means, standart deviation and range. The Student's t test for unpaired data was used to compare means. Changes in plaque size, penile curvature, PDQ, and IIEF- 5 scores from baseline to end of therapy were analyzed using ranked one-way analysis of variance (ANOVA). P values less than 0.05 were considered statistically significant.

\section{RESULTS}

Of the 61 patients who were admitted to our clinic for Peyronie's disease, 48 patients who met the inclusion criteria were included in the study. All patients completed the 8-week treatment protocol. No patients were excluded from the study because no serious side effects or worsening of symptoms were observed during the protocol. The mean age was $61.1(43-78)$. The average time from the onset of complaints was 3.4 months (0-9). Curvature was dorsal in $19(39.6 \%)$ patients, ventral in $5(10.4 \%)$ patients, lateral in $12(25 \%)$ patients, and dorsolateral in $12(25 \%)$ patients. The average plaque size before treatment was $13.6 \mathrm{~mm}$ (7.1-16.8), and after treatment, this value decreased to $10.8 \mathrm{~mm}(4.3-14.6)(\mathrm{p}=0.025)$ (Figure 1). In $35(72.9 \%)$ of the 48 patients, a decrease in plaque size was seen after treatment. Plaque size did not increase in for any of the patients after treatment. The mean curvature angle was 55.7 degrees (35-85) before treatment, whereas this value decreased to 52.5 degrees (33-85) after treatment. In 8 (16.7\%) of the 48 patients, the curvature angle was reduced, but this decrease was not statistically significant (p:0.07) (Figure 2). In five patients (10.4\%), curvature angle was increased. This increase was no more than 10 degrees. The changes in plaque size and curvature angle did not show any statistically significant difference with respect to curvature direction $(\mathrm{p}=$ 0.145). No local or systemic side effects or complications were observed during or after drug administration, excluding pain resulting from the injection of the drug.

The average IIEF-5 score before treatment was 18.1 (10-25). This score increased to 20.2 (10-25) after treatment. However, this rise was not statistically significant $(\mathrm{p}=0.069)$. The PDQS score was $12.3(4-18)$ on average, and this value decreased significantly to $8.9(0-14)$ after treatment $(\mathrm{p}=0.001)$. The PDQP score average was 19.1 (12-26) before treatment and significantly decreased to $9.6(0-21)$ after treatment (p $<0.001)$. Finally, the PDQB score decreased significantly after treatment, as did the other scores. Before treatment, this value was $6.2(4-12)$, and after treatment, it was $4.4(2-10)(\mathrm{p}=0.045)$ (Figure 3$)$.

\section{DISCUSSION}

Surgical treatment modalities of Peyronie's disease are continually being explored and increasing in diversity. However, surgical procedures can be performed only in the chronic phase of the disease [4]. In the acute phase in which inflammation is in progress, there are not many treatment methods with proven effectiveness [5]. Today, the most effective acute phase treatment is provided with collagenase clostridium histolyticum. This is the only treatment method licensed by the FDA. The effectiveness of Collagenase clostridium histolyticum has been proven by many recent studies [14-16]. However, especially in developing countries, using this agent can be very costly and the procurement of the drug can be difficult.

There are many studies about the etiology and physiopathology of Peyronie's disease in the literature. These studies generally focus on genetic, traumatic, and inflammatory processes [17-19]. However, regardless of the underlying cause, the inflammatory process that occurs later in the disease forms the basis of plaque formation. In the course of inflammation, Peyronie's disease occurs due to the production of inducible nitric oxide synthase (iNOS), which leads to an increase of nitric oxide and a corresponding increase in production of peroxynitrite anion. These processes result in the proliferation of fibroblasts and myo-fibroblasts and excessive production of collagen between the layers of the tunica albuginea, which is called penile plaque [20]. 
Corticosteroids are frequently considered to be an acute phase treatment modality of Peyronie's disease due to their intense anti-inflammatory effects [21]. However, few studies have investigated the intralesional use of steroids. Of those that have, positive results were obtained with intralesional steroid use in terms of improving penile curvature angle, plaque size, and penile pain and discomfort during erection [22,23 However, the use of these drugs has not become widespread, and this is largely due to the systemic side effects caused by the use of high doses of steroids and the local side effects that appear around the area of application. As far as we know, this study is the first research to consider low dose methylprednisolone in the treatment of Peyronie's Disease. In our study, statistically significant improvements were found for this treatment, especially in plaque size, severity of symptoms, and penile pain and discomfort during erection. We believe that the use of the drug in a low dose of $40 \mathrm{mg}$ and with one-week intervals between injections would prevent the expected complications of steroids.

PDQ is a questionnaire that has been used many times to investigate the effectiveness of treatment methods used in Peyronie's disease. Its reliability and effectiveness have been proven [24,25]. It is a scale that assesses the patient's perspective on the disease, not just in the physical sense, but also in the psychosexual perception. It was observed that the complaint of pain-one of the typical findings of Peyronie's disease, which disturbs the patient and occurs during erection-decreased significantly after methylprednisolone treatment. Naturally, the sexual performance of patients will improve with the reduction of this complaint. Our study showed an increase in IIEF-5 scores after treatment; however, this increase was not statistically significant. This may be due to the low number of patients in our study. Another reason could be that basal IIEF-5 values are already high, so there may be no significant increase after treatment.

\section{Study Limitations}

The main limitation of the current study was the low number of patients. Additionally, it is not a placebocontrolled randomized study. However, we believe that the results obtained from our study are likely to be replicated in comprehensive double-blind randomized placebo-controlled studies that should be done in the future. One of the methodological limitations was the preferred method of plaque size measuring. Using ultrasound for measuring the plaque size is easy but not reliable because the variable and subjective measurement depends on the person who performs the ultrasound. In order to minimize this subjectivity, all ultrasound procedures were performed by same clinician. Finally, the short follow up time after treatment protocol negatively affected the expected change in IIEF scores because IIEF is made to evaluate erectile function in the past six months.

\section{Conclusion}

The etiology of Peyronie's disease has not been fully elucidated, but the inflammatory process has an important place in the disease's physiopathology. The fact that the only drug that is licensed for the acute phase of the disease is collagenase clostridium histolyticum, and that the effectiveness of other previously-attempted methods has not been fully proven, makes us predict that new treatment modalities will be investigated in the future as well. Our study shows that intralesional usage of low dose methylprednisolone is effective for improving symptoms and signs during the active period of Peyronie's disease. Thus, it may lead to a more progressive transition of the disease from the acute phase to the chronic phase, resulting in less penile deformity and less subsequent complicated correctional surgeries for affected patients.

\section{DISCLOSURE STATEMENT}

Authors declare no conflict of interest

\section{REFERENCES}

[1] Abdel Raheem A, Johnson M, Ralph D, et al. Collagenase clostridium histolyticum: a novel medical treatment for Peyronie's disease. Minerva Urol Nefrol. 2018;70:380-385.

[2] Lipshultz LI, Goldstein I, Seftel AD, et al. Clinical efficacy of collagenase Clostridium histolyticum in the treatment of Peyronie's disease by subgroup: results from two large, double-blind, randomized, placebo- 
controlled, phase III studies. BJU International. 2015;116:650-656.

[3] Schwarzer U, Sommer F, Klotz T, et al. The prevalence of Peyronie's disease: results of a large survey. BJU Int. 2001;88:727-730.

[4] Capoccia E, Levine LA. Contemporary Review of Peyronie's Disease Treatment. Curr Urol Rep. 2018;19:51.

[5] Tsambarlis P, Levine LA. Nonsurgical management of Peyronie's disease. Nat Rev Urol. 2019;16:172-186.

[6] Talib RA, Ibrahim MA, Cangüven Ö. Nonsurgical treatment options in Peyronie's Disease: 2016 update. Turk J Urol. 2016;42:217-223.

[7] Abdel Raheem A, Capece M, Kalejaiye O, et al. Safety and effectiveness of collagenase clostridium histolyticum in the treatment of Peyronie's disease using a new modified shortened protocol. BJU Int. 2017;120:717-723.

[8] Yan S, Yap T, Minhas S. Collagenase clostridium histolyticum intralesional injections for the treatment of Peyronie's disease: a safety profile. Transl Androl Urol. 2017;6:123-126.

[9] Bazzi M, Jamil ML, Dabaja AA. Outcomes of Surgery in Peyronie's Disease Following Intralesional Collagenase Clostridium Histolyticum Injections. Curr Urol Rep. 2019;20:42.

[10] Mehdinasab SA, Alemohammad SA. Methylprednisolone acetate injection plus casting versus casting alone for the treatment of de Quervain's tenosynovitis. Arch Iran Med. 2010;13:270-274.

[11] Evaniew N, Belley-Côté EP, Fallah N, et al. Methylprednisolone for the Treatment of Patients with Acute Spinal Cord Injuries: A Systematic Review and Meta-Analysis. J Neurotrauma. 2016;33:468-481.

[12] Turunç T, Deveci S, Güvel S, et al. The assessment of turkish validation with 5 question version of international index of erectile function (IIEF-5). Turkish Journal of Urology. 2007;33:45-49.

[13] Hellstrom Wayne J.G., Feldman Robert, Rosen Raymond C., et al. Bother and Distress Associated with Peyronie's Disease: Validation of the Peyronie's Disease Questionnaire. Journal of Urology. 2013;190:627-634.

[14] Capece M, Cocci A, Russo G, et al. Collagenase clostridium histolyticum for the treatment of Peyronie's disease: a prospective Italian multicentric study. Andrology. 2018;6:564-567.

[15] Cocci A, Cito G, Urzì D, et al. Sildenafil $25 \mathrm{mg}$ ODT + Collagenase Clostridium hystoliticum vs Collagenase Clostridium hystoliticum Alone for the Management of Peyronie's Disease: A Matched-Pair Comparison Analysis. J Sex Med. 2018;15:1472-1477.

[16] Ralph DJ, Abdel Raheem A, Liu G. Treatment of Peyronie's Disease With Collagenase Clostridium histolyticum and Vacuum Therapy: A Randomized, Open-Label Pilot Study. J Sex Med. 2017;14:1430-1437.

[17] Dam E-JPM ten, Driel MF van, Jong IJ de, et al. Glimpses into the molecular pathogenesis of Peyronie's disease. The Aging Male. 2019;0:1-9.

[18] Rose AFD, Mantica G, Bocca B, et al. Supporting the role of penile trauma and micro-trauma in the etiology of Peyronie's disease. Prospective observational study using the electronic microscope to examine two types of plaques. The Aging Male. 2019;0:1-6.

[19] Gunes M, Aslan R, Eryllmaz R, et al. Levels of serum trace elements in patients with Peyronie. The Aging Male. 2018;0:1-4.

[20] Paulis G, Brancato T. Inflammatory mechanisms and oxidative stress in Peyronie's disease: therapeutic "rationale" and related emerging treatment strategies. Inflamm Allergy Drug Targets. 2012;11:48-57.

[21] Taylor FL, Levine LA. Peyronie's Disease. Urol Clin North Am. 2007;34:517-534, vi. 
[22] Bodner H, Howard AH, Kaplan JH. Peyronie's disease; cortisone-hyaluronidase-hydrocortisone therapy. Trans West Sect Am Urol Assoc. 1953;20:32-35.

[23] Winter CC, Khanna R. Peyronie's disease: results with dermo-jet injection of dexamethasone. J Urol. 1975;114:898-900.

[24] Serefoglu EC, Smith TM, Kaufman GJ, et al. Factors Associated With Erectile Dysfunction and the Peyronie's Disease Questionnaire in Patients With Peyronie Disease. Urology. 2017;107:155-160.

[25] Coyne KS, Currie BM, Thompson CL, et al. The test-retest reliability of the Peyronie's disease questionnaire. J Sex Med. 2015;12:543-548.

Table 1. Characteristics of the patients.

\begin{tabular}{lllll}
\hline & Mean & Min & Max & SD $( \pm)$ \\
\hline Age & 61.1 & 43 & 78 & 11.8 \\
Disease duration (months) & 3.4 & 0 & 9 & 2.3 \\
Baseline curvature $\left(^{\circ}\right)$ & 55.7 & 35 & 85 & 24.3 \\
Baseline plaque size $(\mathrm{mm})$ & 13.6 & 7.1 & 16.8 & 3.7 \\
\hline
\end{tabular}

Table 2. Baseline values according to direction of the curvature.

\begin{tabular}{|c|c|c|c|c|c|}
\hline & Dorsal (n:19) & Ventral (n:5) & Lateral (n:12) & $\begin{array}{l}\text { Dorsolateral } \\
(\mathrm{n}: 12)\end{array}$ & $\mathrm{p}$ \\
\hline $\begin{array}{l}\text { Age } \\
(\text { mean } \pm \text { SD })\end{array}$ & $63.3 \pm 12.1$ & $53.2 \pm 5.4$ & $65.3 \pm 7.8$ & $56.9 \pm 11.1$ & 0.135 \\
\hline $\begin{array}{l}\text { Plaque Size } \\
(\mathrm{mm}) \\
(\mathrm{mean} \pm \mathrm{SD})\end{array}$ & $11.9 \pm 3.1$ & $15.6 \pm 1.2$ & $15.9 \pm 2.4$ & $13.3 \pm 2.3$ & 0.065 \\
\hline $\begin{array}{l}\text { Angle of } \\
\text { Curvature }\left(^{\circ}\right) \\
(\text { mean } \pm \mathrm{SD})\end{array}$ & $50.4 \pm 12.9$ & $62 \pm 8.4$ & $54.5 \pm 10.1$ & $62.7 \pm 20.5$ & 0.045 \\
\hline
\end{tabular}

\section{Figure Legends}

Figure 1. Improvement in plaque size after treatment.

Figure 2. Improvement in curvature angle after treatment.

Figure 3. Changes in questionnaire scores after treatment. IIEF-5: Five question version of International Index of Erectile Fuction; PDQS: Peyronie's Disease Questionnaire Symptom Severity; PDQP: Peyronie's Disease Questionnaire Penile Pain; PDQB: Peyronie's Disease Questionnaire Bother/Discomfort. 
p:0.025

13.6

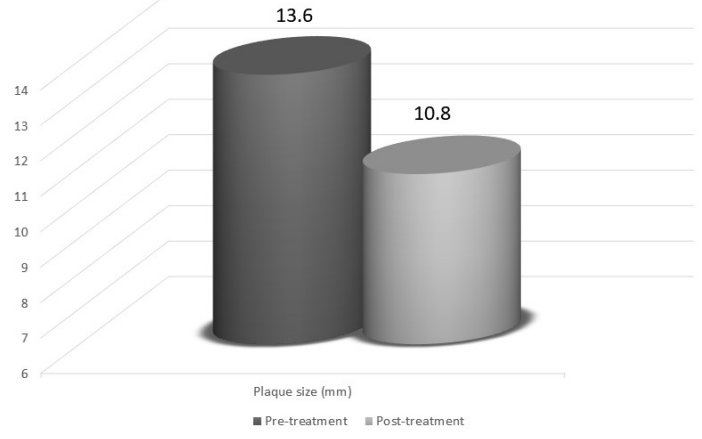

p:0.07

55.2

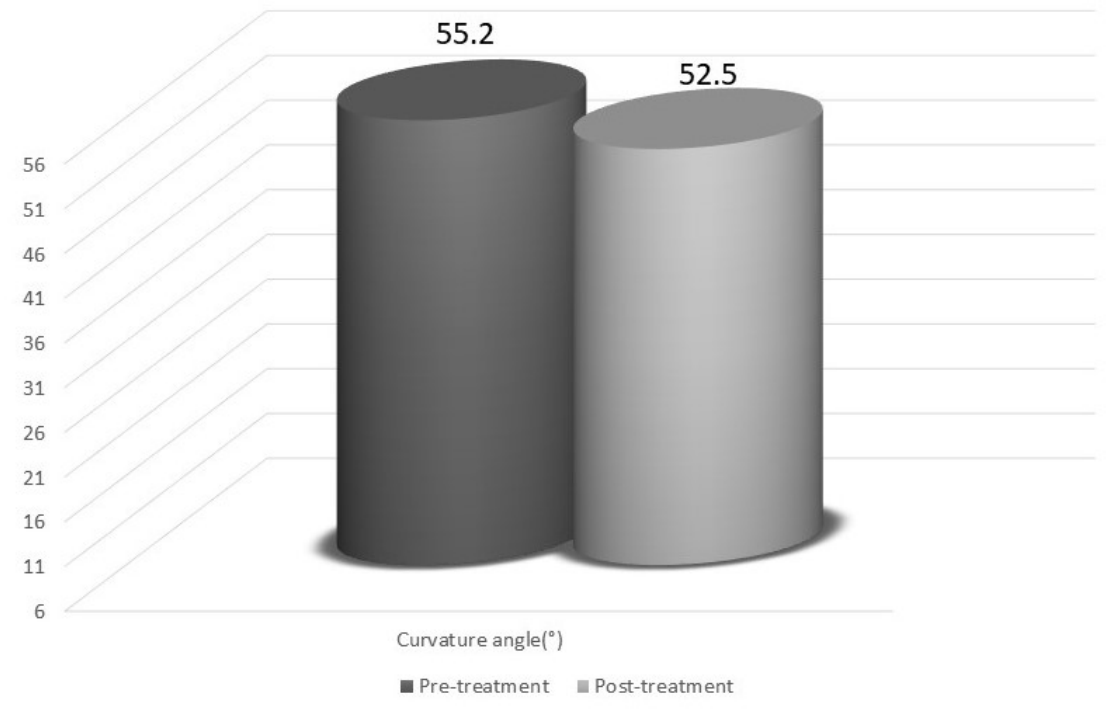




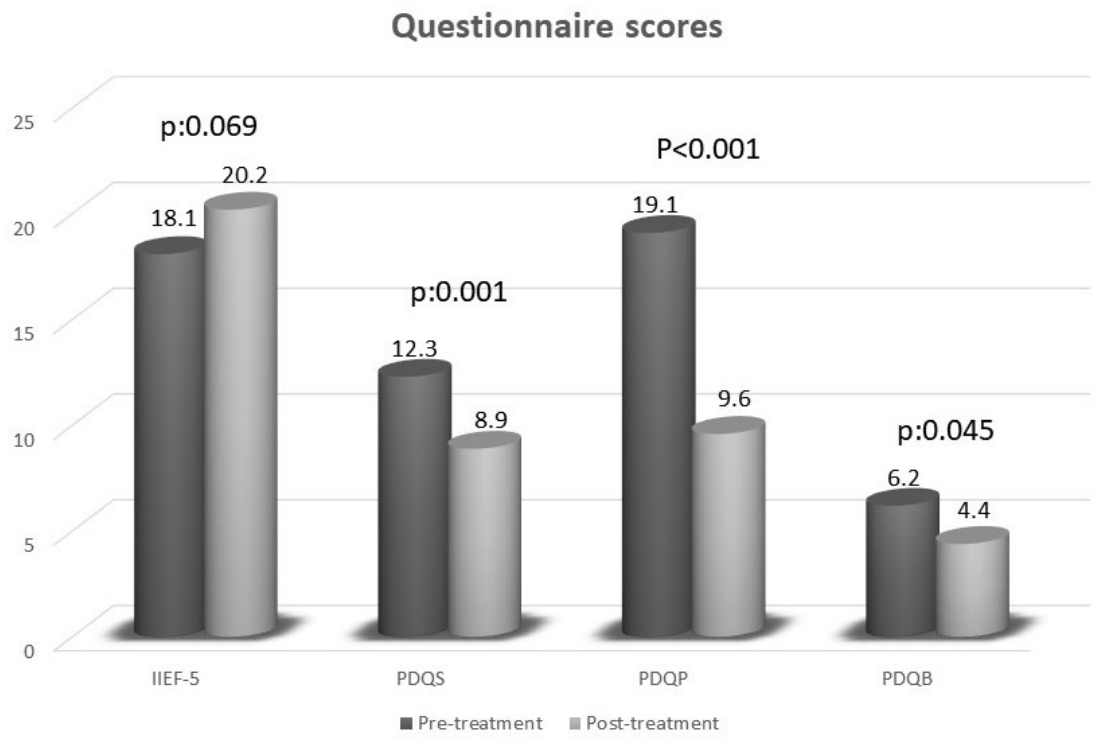

